\title{
High-Resolution X-ray Fluorescence and Excitation Spectroscopy of Metalloproteins
}

\author{
Xin Wang, ${ }^{a, b}$ Melissa M. Grush, ${ }^{a, b}$ Andrew G. Froeschner ${ }^{a}$ and \\ Stephen P. Cramera,b \\ ${ }^{a}$ Department of Applied Science, University of California, Davis, CA 95616, USA, and \\ ${ }^{b}$ Energy and Environment Division, Lawrence Berkeley National Laboratory, Berkeley, \\ CA 94720, USA. E-mail: cramer@seka.ucdavis.edu
}

(Received 8 July 1996; accepted 16 December 1996)

\begin{abstract}
A spectrograph has been developed with sufficient efficiency to make high-resolution fluorescence experiments on metalloproteins possible. The resolution of this spectrometer can reach $0.45 \mathrm{eV}$ at $7.1 \mathrm{keV}$ emission energy. The focus images of this multiple curved-crystal array spectrometer are presented. The chemical sensitivity of $K / 3$ emission spectra can be used to identify chemical states, and the spin-polarized near-edge structure provides a new measure of the spin density. The high-resolution fluorescence metalloprotein studies should become routine with third-generation synchrotron facilities, and the strength of both site and spin selectivity should complement the structural information from other spectroscopies.
\end{abstract}

Keywords: $X$-ray fluorescence; $K \beta$ emission; spin polarization; metalloproteins.

\section{Introduction}

The combined application of X-ray absorption and emission spectroscopy to chemical and physical problems offers unique advantages over simple X-ray absorption or emission measurements (Åberg \& Tulkki, 1985; Cowan, 1994). This rapidly developing field is variously known as resonant X-ray emission (RXES) (Mori, Kayanuma \& Kotani, 1991), resonant X-ray fluorescence (RXFS) (Skytt, Guo, Wassdahl \& Nordgren, 1995), resonant X-ray inelastic scattering (RXIS, RIXS or REXS) (de Groot, 1996; Krisch et al., 1995), or X-ray resonant scattering or X-ray resonance Raman scattering (XRRS) (Carra, Fabrizio \& Thole, 1995; Udagawa, Hayashi, Tohji \& Mizushima, 1994). Regardless of the nomenclature, one essential part of the experiment is high-resolution monochromatization of both the resonant $\mathrm{X}$-ray excitation beam and of the scattered or emitted radiation. The first X-ray resonance Raman scattering was observed by Sparks (1974) with monochromatic $\mathrm{Cu} \mathrm{K \alpha}$ radiation incident on various metallic targets. The first synchrotron radiation resonance Raman scattering experiment was carried out by Eisenberger and coworkers (Eisenberger, Platzman \& Winick, 1976a,b), who studied the effect near the $K$ edge of metallic $\mathrm{Cu}$. Development of ever-brighter synchrotron radiation sources (Schlacter, 1994; Winick, 1995) has dramatically improved the sensitivity of these double-monochromator experiments. Monitoring the emission has improved from low-resolution measurements using solid-state detectors to high-resolution experiments with curved-crystal (Stojanoff et al., 1992) or grating (Nordgren et al., 1989) spectrometers.

Chemical effects on the energies and shapes of X-ray fluorescence lines have been known since the 1920s (Lindh
\& Lundquist, 1924). These shifts can be used to identify oxidation and spin states (Ekstig, Källne, Noreland \& Manne, 1970; Peng, de Groot et al., 1994). Shifts due to oxidationstate changes can be used to conduct site-selective XANES or EXAFS measurements (Grush, Christou, Hämäläinen \& Cramer, 1995). Shifts due to spin-state changes can also be used for this purpose (Wang \& Cramer, 1997). The spin sensitivity of the emission spectrum can also be used to generate spin-selective XANES (Hämäläinen et al., 1992; Peng, Wang, Randall, Moore \& Cramer, 1994; Wang, Randall, Peng \& Cramer, 1995) or spinpolarized EXAFS measurements (SPEXAFS). The resultant SPEXAFS effects at the $K$ edge (Grush, Horne \& Cramer, 1997; Wang, Cramer et al., 1997) are 50-100 times stronger than those observed by X-ray magnetic circular dichroism measurements (Schütz et al., 1987; Kobayashi et al., 1995). Dramatic line-sharpening effects in X-ray scattering measurements allow observation of structure in excitation spectra that is normally unobservable (Hämäläinen, Siddons, Hastings \& Berman, 1991).

High-resolution fluorescence and scattering experiments on the metal clusters in proteins complement the electronic and structural information available from X-ray crystallography, EXAFS, and other spectroscopies. Because the metals are present at low concentration (30-1000 p.p.m.), the efficiency of the collecting spectrograph is critical to the feasibility of such experiments. In this paper, we describe a curved-crystal array for collecting X-ray emission and excitation spectra. This device has an energy resolution of $10^{-4}(\Delta E / E)$ and collects sufficient solid angle to make metalloprotein experiments possible. We report Fe emission and excitation spectra of Pyrococcus furiosus rubredoxin that demonstrate the feasibility of applying these 
techniques to metalloproteins. These results also illustrate the spin-dependent electronic information obtainable. The significance of X-ray photoreduction is illustrated with preliminary results on the Mn in Photosystem II. The potential for further improvements and prospects for biological applications are discussed.

\section{Experimental apparatus and procedures}

\subsection{High-resolution fluorescence spectrometer}

In order to study dilute systems such as metalloproteins, a multiple crystal array spectrometer was developed which captures a larger solid angle than previously possible (Fig. 1). The incident beam is focused on the sample using the beamline optics. An array of spherically bent crystals on intersecting Rowland circles then diffracts the scatter or fluorescence back to a common spot on the detector. Because the diffraction occurs in near-backscattering Johann geometry on an $850 \mathrm{~mm}$-diameter Rowland circle, very high energy resolution $\left(\Delta E / E=10^{-4}\right)$ can be obtained. An emission spectrum can be obtained by rotating the analyzer crystals while moving the detector (in this case, an NaI scintillation counter) simultaneously to track the diffracted beam. Alternatively, a position-sensitive detector can be used, eliminating the necessity of having a mobile detector.

The previous spectrometer with a single analyzer (Stojanoff et al., 1992) used a mechanical crystal-bending apparatus (Schulke \& Nagasawa, 1984). However, the extra space occupied by crystal benders prevents efficient packing of multiple crystals into an array. We therefore switched to 'form-bent' crystals, each glued onto a spherically ground concave glass form with an $850 \mathrm{~mm}$ radius. For $\mathrm{Fe} K \beta$ emission, the $78^{\circ}$ Bragg angle obtained with $\operatorname{Ge}(620)$ crystals gives $0.45 \mathrm{eV}$ resolution at $7.1 \mathrm{keV}$ (Brennan \& Cowan, 1992), while the $\mathrm{Si}(440)$ reflection at $83.4^{\circ}$ used for $\mathrm{Mn} K \beta$ yields $0.35 \mathrm{eV}$ resolution at $6.8 \mathrm{keV}$ (Stojanoff et al., 1992). Each 'form-bent' crystal is placed in a circular opening machined from an aluminium plate and held in place with a set screw. The crystal holder is mounted on a computer-controlled Huber rotation stage which sets the diffraction angle $(\theta)$. Another goniometer at the bottom drives rotation in the horizontal plane $(\chi)$. The six crystals are placed as close together as possible (Fig. 1). The solid angle collected by each crystal is $0.28 \mathrm{msr}$.

Reduction of air absorption is essential because of the long paths taken by the fluorescence X-rays in this experiment. A plastic (PVC) vacuum tank was built to occupy most of the path between sample, crystals and detector. $50 \mu \mathrm{m}$ Kapton windows were used on both ends of the tank and a vacuum of approximately 6 Torr was obtained. Air absorption was further minimized by additional helium paths between the vacuum tank and detector, between the crystals and the tank, and between the beamline and the sample. The overall transmitted intensity of this spectrometer is 0.70 at $6.8 \mathrm{keV}$, the result of $19 \mu \mathrm{m}$ polypropylene, $150 \mu \mathrm{m}$ Kapton, $5.1 \times 10^{4} \mu \mathrm{m}$ air, and $7.1 \times 10^{5} \mu \mathrm{m}$ helium in the beam path. A Balzers He refrigerator with a $7 \mathrm{~K}$ copper cold finger was used to keep the sample cold and reduce $\mathrm{X}$-ray damage.

\subsection{Sample preparation and data collection}

$\left(\mathrm{NEt}_{4}\right)\left[\mathrm{FeCl}_{4}\right]$ (Gill, 1961) was a gift from Professor Larry Que. Rubredoxin from Pyrococcus furiosus was prepared by published procedures (Blake et al., 1991) and kindly donated by Professor Michael Adams. The concentration of the protein was $22.5 \mathrm{mg} \mathrm{ml}^{-1}$, corresponding to an Fe concentration of $\sim 225$ p.p.m. A Photosystem II sample originally poised in the $S_{1}$ state (Ghanotakis, Demetriou \& Yocum, 1987) was generously provided by Professor James Penner-Hahn.

$\mathrm{Fe}$ and $\mathrm{Mn} K \beta$ spectra were recorded on the $\mathrm{X}-25$ wiggler beamline at Brookhaven National Laboratory (Berman, Hastings, Oversluizen \& Woodle, 1992) with the spectrometer described above. The synchrotron radiation excitation beam was monochromated using a pair of $\operatorname{Si}(220)$ crystals and focused with a toroidal mirror to a $0.5 \times 0.8 \mathrm{~mm}$ spot. Mn $K \beta$ spectra and $\mathrm{Fe} \mathrm{X}$-ray images were also recorded on beamline VI-2 at Stanford Synchrotron Radiation Lab-
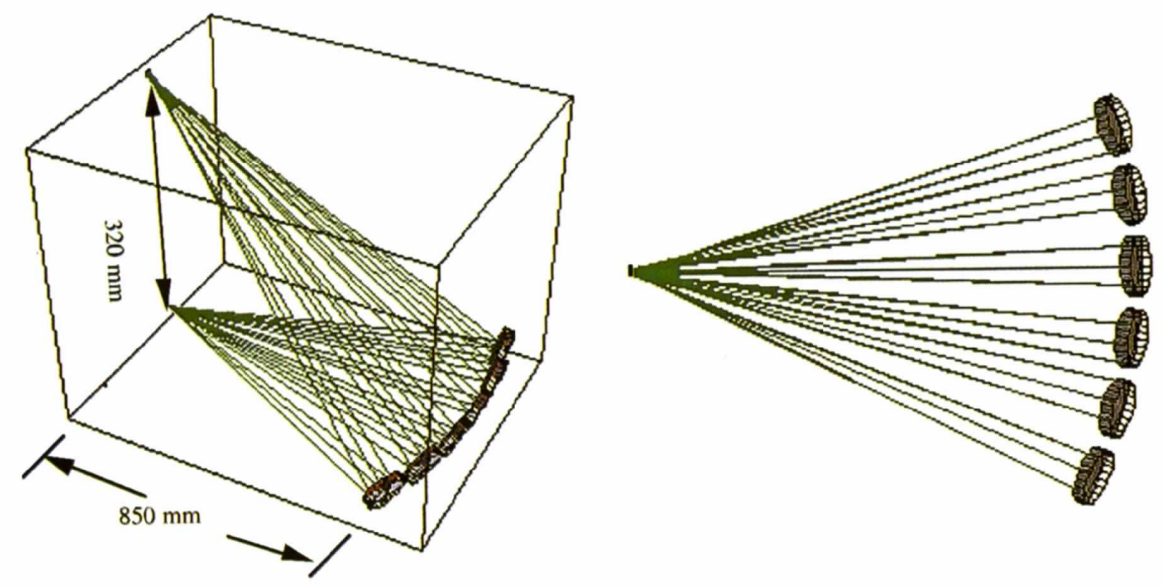

Figure 1

Schematic diagrams of the crystal array used for metalloprotein experiments: side view (left) and top view (right). 
oratory (Hoyer et al., 1983) with an $\mathrm{Si}(111)$ excitation monochromator and the same fluorescence spectrometer.

The Fe spectra were calibrated by assigning the $K \beta$ peak of $\left(\mathrm{NEt}_{4}\right)\left[\mathrm{FeCl}_{4}\right]$ to $7058.0 \mathrm{eV}$ (Peng, Wang et al., 1994); Mn emission spectra used a value of $6491.7 \mathrm{eV}$ for the main $K \beta$ peak of $\mathrm{MnF}_{2}$ (Grush et al., 1995). The excitation energy was calibrated by reference to the first inflection point of Fe or Mn metal, 7111.2 and $6539.0 \mathrm{eV}$, respectively (Williams, 1986).

\section{Results and discussion}

\subsection{Spectrometer focus quality}

To evaluate the combined focus in our array spectrometer, images were recorded with a two-dimensional position-sensitive detector (Smith \& Yu, 1995). This gasproportional chamber has an active area of $10 \times 2 \mathrm{~cm}$, with a position resolution of approximately $200 \mu \mathrm{m}$ at $7 \mathrm{keV}$. Using this detector, the image of the fluorescence focus was recorded separately for each individual analyzer crystal, as well as for three crystals simultaneously.

The images of the diffracted beams collected from the three crystals are shown in Fig. 2. Contours were drawn for the beam from each crystal while the other crystals were blocked. The finite source size of the excitation beam on the $\left(\mathrm{NEt}_{4}\right)\left[\mathrm{FeCl}_{4}\right]$ sample $(1 \mathrm{~mm}$ vertical $\times 3 \mathrm{~mm}$ horizontal $)$ and the spherical aberration of the 'form-bent' crystals contribute to the size and shape of the diffracted beam.

\section{$1 \mathrm{~cm}$}

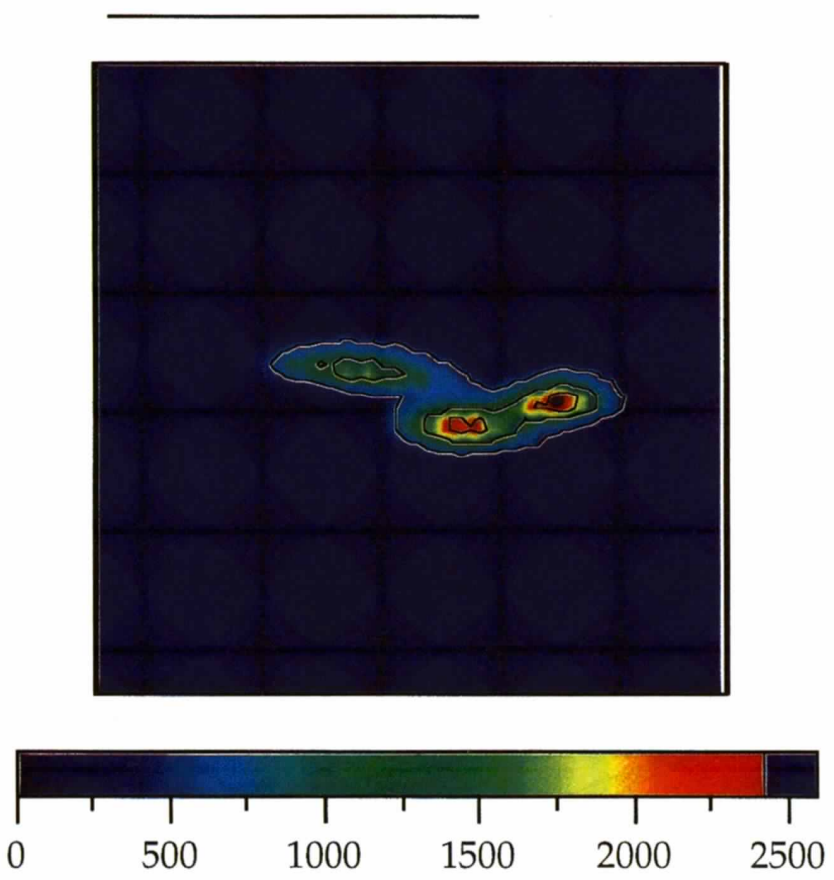

Figure 2

Illustration of the focus image achieved with three crystals simultaneously. The label at the bottom shows the photon counts corresponding to a given color. The horizontal and vertical axes in the picture are also the horizontal and vertical axes, respectively, for the position-sensitive detector.
In this geometry, the vertical axis is the energy-dispersive direction on each Rowland circle. The individual beams focus to $1.5 \times 2.1 \mathrm{~mm}$ (FWHM) for each crystal, and the combined beams stay within a $3.8 \times 8.5 \mathrm{~mm}$ region. Thus, a small $(4 \times 9 \mathrm{~mm})$ slit can be used to reduce the background from undesirable scattered X-rays.

\subsection{Comparison of 'form-bent' and mechanically bent crystals}

In Fig. 3, we compare the emission spectrum of $\left(\mathrm{NEt}_{4}\right)\left[\mathrm{FeCl}_{4}\right]$ using a mechanically bent $\mathrm{Ge}(620)$ crystal with that using a 'form-bent' $\mathrm{Ge}(620)$ crystal. Even after the focus of the mechanically bent crystal was optimized using $\mathrm{Fe} K \beta$ fluorescence, the Fe emission spectrum was broader than that observed with the 'form-bent' crystal. The observed FWHM of the emission spectrum improved from $7.2 \mathrm{eV}$ to $5.3 \mathrm{eV}$, increasing the intensity at the $K \beta$ peak by $25 \%$. We also found it difficult to bend $\mathrm{Ge}$ crystals to the desired radius without frequent breakage. We note, however, that bending $\mathrm{Si}(440)$ crystals was less problematic, and improved resolution was not obtained with 'form-bent' $\mathrm{Si}(440)$ crystals.

\subsection{Count rate for an Fe model compound}

A typical count rate at the $\mathrm{Fe} K \beta$ peak for pure $\left(\mathrm{NEt}_{4}\right)\left[\mathrm{FeCl}_{4}\right]$ with three 'form-bent' crystals was $\sim 3000$ photons s $^{-1}$ with a background of only $\sim 4$ counts $\mathrm{s}^{-1}$. With an incident flux of $1 \times 10^{12}$ photons $\mathrm{s}^{-1}$, it is a worthwhile exercise to determine where the photons

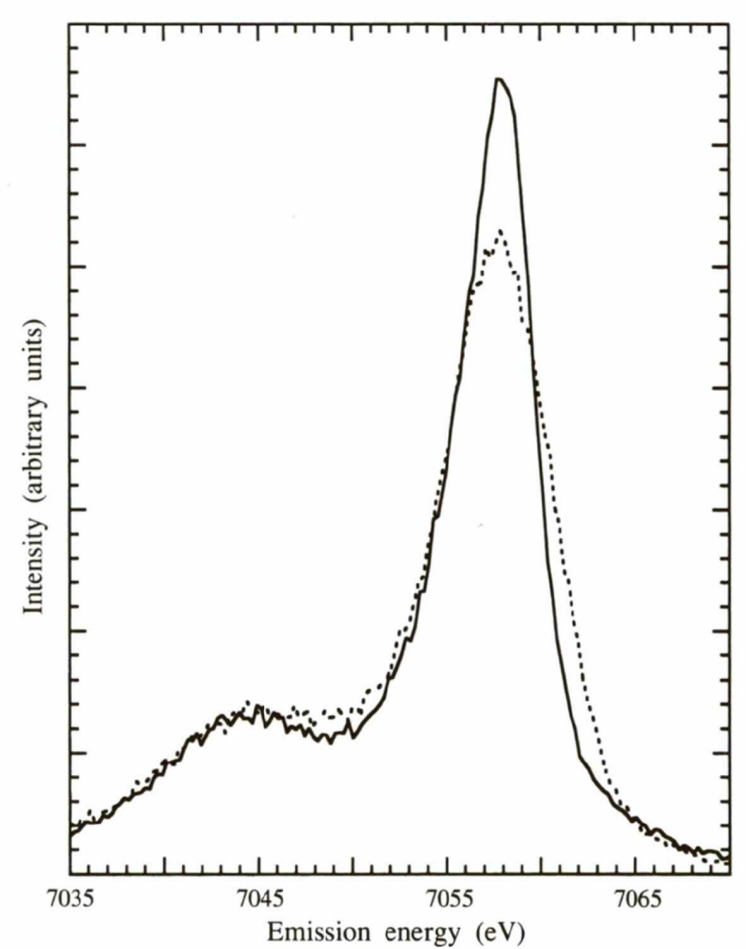

Figure 3

Improved resolution achieved with a form-bent $\mathrm{Ge}(620)$ crystal (solid line) as opposed to a mechanically bent crystal (dotted line), illustrated with the $K / 3$ emission spectrum of $\left(\mathrm{NEt}_{4}\right)\left[\mathrm{FeCl}_{4}\right]$. 
have gone. If we assume that $53 \%$ of the incident X-rays are absorbed by $\mathrm{Fe}$ [calculated using absorption coefficients as tabulated in Marenkov (1994)], a total fluorescence yield of $34 \%$ (Krause, 1979), a $K \beta / K \alpha$ quantum yield ratio of 0.133 , and $75 \%$ reabsorption of the emitted radiation, then approximately $5.1 \times 10^{9}$ photons $^{-1}$ are emitted into $4 \pi$ steradians. Since the spectrograph only intercepts $6.7 \times 10^{-5}$ of the full solid angle and the windows and air paths absorb $50 \%$ of the $\mathrm{X}$-rays, we can expect at most a count rate of $1.7 \times 10^{5}$ photons $\mathrm{s}^{-1}$. (The most recent version of this spectrometer includes thinner Kapton windows and a smaller air path, resulting in a transmission of $70 \%$, as stated earlier in this manuscript, rather than the $50 \%$ used in this efficiency calculation.) This calculated count rate is still greater than 50 times that observed. However, as the $K \beta$ emission is distributed over more than $20 \mathrm{eV}$, while the rocking curve width of the $\mathrm{Ge}$ crystal ( $71 \mu \mathrm{rad})$ (Brennan \& Cowan, 1992; Zachariasen, 1945) corresponds to approximately $0.33 \mathrm{eV}$ resolution, we conclude that the remaining 'lost' photons are a consequence of the high resolution of this spectrometer. For certain experiments where only resolution of $K \beta$ and $K \beta^{\prime}$ features is necessary, an order of magnitude in count rate could be recaptured with a lower-resolution instrument.

\subsection{Fe K's emission spectrum of rubredoxin}

In Fig. 4 , the $K \beta$ emission spectrum (measured with an excitation energy of $7200 \mathrm{eV}$ ) of reduced Pyrococcus furiosus rubredoxin is compared with that from $\left(\mathrm{NEt}_{4}\right)\left[\mathrm{FeCl}_{4}\right]$. Both the protein and the model complex are high-spin

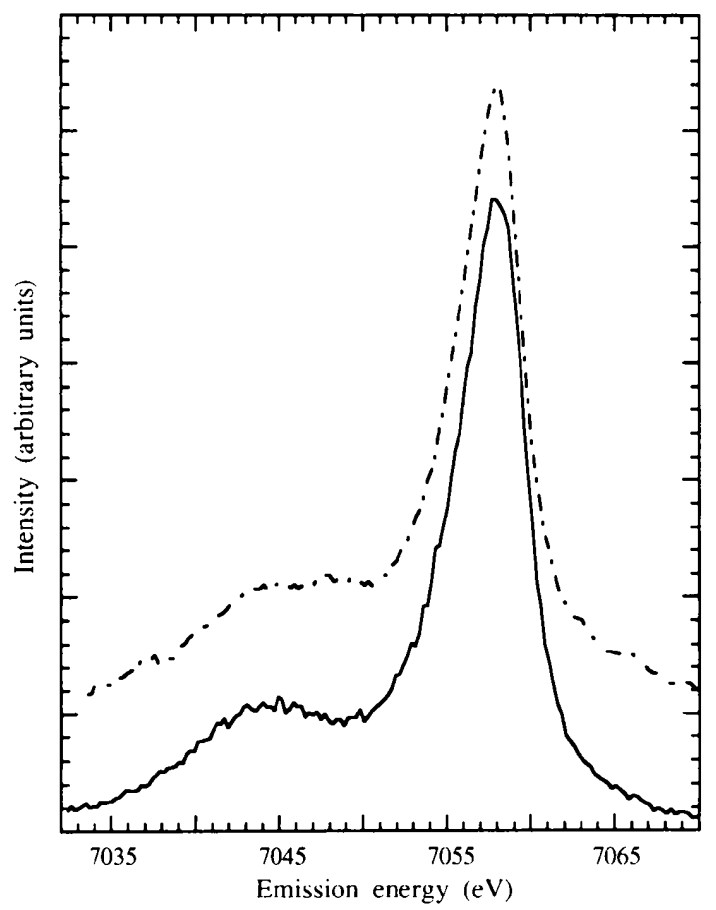

Figure 4

Experimental Fe $K, 3$ spectrum of rubredoxin (dotted line) compared with the tetrahedral model complex $\left(\mathrm{NEt}_{4}\right)\left[\mathrm{FeCl}_{4}\right]$ (solid line).
Fe compounds with approximately tetrahedral symmetry. The $K / 3$ emission spectra are split into two regions, the main peak $(K / 3)$ around $7057.9 \mathrm{eV}$ and a satellite $\left(K 3^{\prime}\right)$ at lower energy. The $K / 3$ regions are very similar in the two spectra, but the position and intensity of the satellites differ. For $\left(\mathrm{NEt}_{4}\right)\left[\mathrm{FeCl}_{4}\right]$, the $K \beta^{\prime}$ satellite is stronger and better resolved, with a $14 \mathrm{eV}$ energy separation from the main peak, while the rubredoxin has a weaker satellite $\sim 13 \mathrm{eV}$ lower in energy than the main peak. The weaker satellite in the protein spectrum may be due in part to the more covalent sulfur ligands as compared to chloride ligands in the model compound. As reviewed by Ekstig et al. (1970), Peng, de Groot et al. (1994) and de Groot, Fontaine, Kao \& Krisch (1994), the splitting of the $K_{3} / 3$ main peak and satellite is due to the $3 p 3 d$ exchange interaction. When $3 d$ electrons are delocalized as in the case of covalent sulfur ligands, the smaller exchange interaction results in a smaller energy separation from the main peak and a weakened satellite. A more detailed analysis of these spectra will be presented elsewhere (Wang, Huang, Adams, Kotani \& Cramer, 1997). The important point is that the $\mathrm{Fe} K 3$ spectrum of rubredoxin demonstrates that there is adequate sensitivity for recording emission spectra of metalloproteins. In many cases, there will be valuable chemical information in these emission spectra. In addition, the spin-polarized nature of $K i 3$ emission allows the measurement of spin-selective XANES.

\subsection{Spin-polarized XANES}

The predominately 'spin-down' versus 'spin-up' nature of the $K /$ and $K \beta^{\prime}$ regions, respectively, allows spinpolarized X-ray excitation spectra to be recorded. The fluorescence from either region can be monitored as a function of incident energy. In Fig. 5, we show the first spin-polarized XANES of a metalloprotein (rubredoxin) recorded in this manner. There are several differences between the spin-up and spin-down spectra. The spin-down edge generally has sharper, better-defined features than the spin-up spectrum. There is a clear double peak at the top of spin-down edge, while the spin-up edge has a single broad peak. Finally, there is a clear pre-edge peak around $7112.6 \mathrm{eV}$ in the spin-down spectrum, which is generally described as a $1 s \rightarrow 3 d$ transition.

The $1 s \rightarrow 3 d$ transition provides a demonstration of the spin-polarized nature of these excitation spectra. In a tetrahedral environment, mixing of $4 p$ and $3 d$ orbitals gives this transition some dipole-allowed character. For high-spin $\mathrm{Fe}^{\| l}$, a $d^{t}$ ion, the electronic configuration can be described as $e_{g}{ }^{3} t_{2 g}{ }^{3}$, and all of the 'spin-up' orbitals are filled. Transitions to the remaining $t_{2 g}$ spin-down levels are allowed, hence the ' $1 s \rightarrow 3 d$ ' transition in the spin-down spectrum. However, since there are no spin-up vacancies, the spin-up spectrum does not show a ' $1 s \rightarrow 3 d$ ' transition. The residual intensity in this region results from resonant Raman scattering (de Groot et al., 1995). A more detailed discussion of the rubredoxin data with theoretical simulations is in preparation (Wang, Huang et al., 1997). 
Ionic samples show clearer definition between the edge and pre-edge regions as compared with more covalent samples with similar geometry. This has been previously illustrated by comparing spin-polarized XANES from $\mathrm{MnO}_{2}$ and $\mathrm{MnF}_{2}$ (Hämäläinen et al., 1992). As a contrast to the relatively covalent rubredoxin case, the spin-polarized XANES of $\mathrm{MnF}_{2}$ are presented in Fig. 6.

\subsection{Photosystem II - a dilute challenge}

A cluster of four $\mathrm{Mn}$ ions forms an essential part of the oxygen-evolving complex of Photosystem II (Tsiotis, McDermott \& Ghanotakis, 1996). As the concentration of $\mathrm{Mn}$ in this system is quite low (typically $\sim 40$ p.p.m. Mn), measurements on Photosystem II illustrate the current sensitivity limit for this technique. The first $\mathrm{Mn} K \beta$ emission spectrum of Photosystem II is shown in Fig. 7. A count

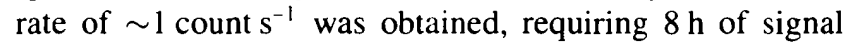
averaging to obtain the spectrum shown here. The beam was moved to a fresh spot on the sample every $2 \mathrm{~h}$. Mn $K / \beta$ emission of Photosystem II poised in various states will be presented elsewhere (Grush, de Marois, Daly, Penner-Hahn \& Cramer, 1997).

Photoreduction during exposure to bright X-ray sources is often a concern during metalloprotein experiments (George, Lowery, Solomon \& Cramer, 1993; George et al., 1992). In recent papers the sensitivity of some

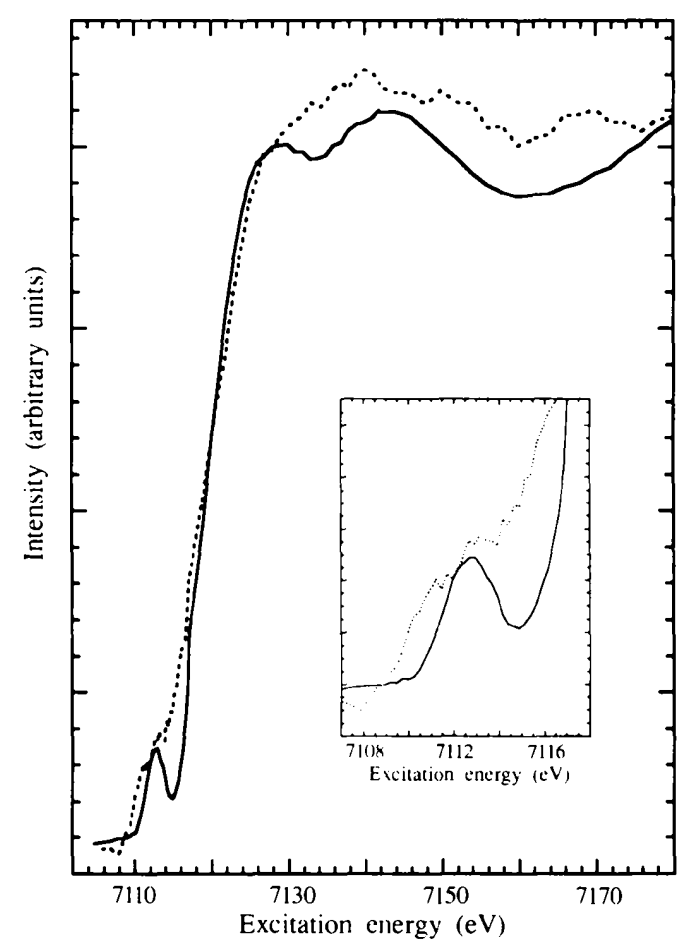

Figure 5

Spin-polarized Fe excitation spectra of rubredoxin. The spin-down transition (solid line) is compared with the spin-up transition (dotted line). The inset is the pre-edge region of both excitation spectra. The spin-down edge was measured by setting the analyzer crystals to the main $K .3$ peak, $7057.9 \mathrm{eV}$, while the spin-up transition was monitored at $7044.9 \mathrm{eV}$. The raw data were smoothed over a 5-point range. metalloproteins has been suggested (Murphy et al., 1995; Debenham et al., 1996). Damage can be reduced by spreading the X-ray beam across a relatively large sample. Conversely, the situation is worse for this experiment, which involves point-to-point focusing.

Comparison of the Photosystem II spectrum with Mn model complexes (Fig. 7) shows that this sample contains predominantly $\mathrm{Mn}^{\mathrm{II}}$. It has been shown using conventional $K$-edge absorption measurements that $\mathrm{Mn}^{\prime \prime}$ is not a component of the resting ' $S_{1}$ ' state (Sauer, Yachandra, Britt \& Klein, 1992), and that the native Mn oxidation states should include $\mathrm{Mn}^{\mathrm{III}}$ (Kirby et al., 1981; Kusonoki, Ono, Matsushita, Oyanagi \& Inoue, 1990; Kusunoki et al., 1991; Penner-Hahn et al., 1990) and possibly $\mathrm{Mn}^{\mathrm{IV}}$ (Yachandra $e t$ al., 1993; Riggs, Mei, Yocum \& Penner-Hahn, 1992). Most likely, this sample has been reduced by electrons produced during exposure to the X-ray beam.

With brighter beamlines, photodamage will become a concern for many systems. Careful monitoring of X-ray spectra for time-related changes as well as performing sample assays before and after X-ray exposure will be necessary in order to ensure the relevance of these $\mathrm{X}$-ray measurements.

\subsection{Future prospects}

High-resolution fluorescence experiments on metalloproteins are clearly starved for photons, and it is worth considering the impact of brighter sources. On thirdgeneration facilities such as the European Synchrotron

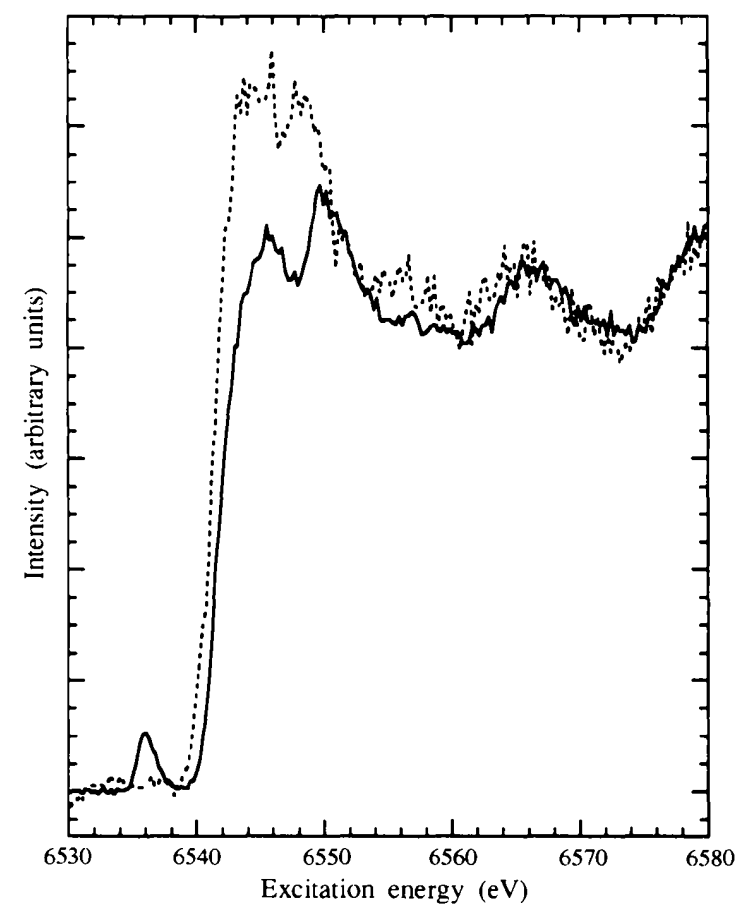

\section{Figure 6}

A demonstration of spin selectivity using $\mathrm{MnF}_{2}$. Mn XANES using $K .3$ detection (solid line) and $K{ }^{\prime} \zeta^{\prime}$ detection (dotted line). The $1 s$ to $3 d$ pre-edge feature is only observed when the outgoing electron is spin down. 
Research Facility (ESRF) and the Advanced Photon Source (APS), high-resolution fluorescence experiments on metalloproteins could become routine. The incoming photon flux on new undulator beamlines will most likely improve by one to two orders of magnitude while still focusing to a sub-millimeter spot. Our current spectrometer has an efficiency of $10^{-7}$ with three crystals. Increasing the solid angle of the analyzers is possible by switching to six $6^{\prime \prime}$-diameter crystals, improving the fluorescence at the detector by 50 -fold, and resulting in a total efficiency of $5 \times 10^{-6}$ \{using the $\left(\mathrm{NEt}_{4}\right)\left[\mathrm{FeCl}_{4}\right]$ complex as an example $\}$. The combined three to four orders of magnitude increase from both incoming photons and spectrometer efficiency could shorten the acquisition time for $K / 3$ emission spectra of metalloproteins to a few minutes, even for $\leq 50$ p.p.m. metal concentrations. However, the bright future of high-resolution metalloprotein experiments is shadowed by the obstacles of photoreduction. With such a high photon brightness, the protein could easily be reduced before a single scan is completed. On-line monitoring through optical or other spectroscopies can be helpful to determine time-related changes. Photoreduction will become the limiting factor for these experiments. One possible solution is to rotate a frozen protein solution during the measurements, requiring much higher homogeneity in sample preparation.

For spin-polarized experiments, the resolution of the curved-crystal spectrometer is much better than necessary. Since the separation between the main peak and satellite

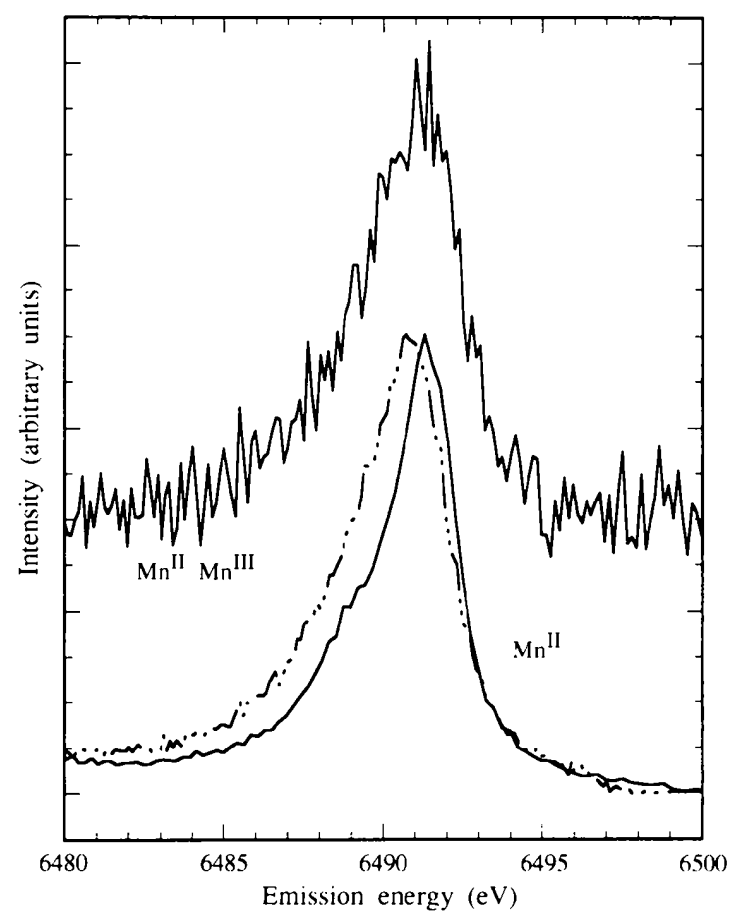

Figure 7

Experimental Mn K,3 emission spectra of photoreduced Photosystem II (top, solid line) compared with $\mathrm{Mn}^{\mathrm{ff}} \mathrm{Cl}_{2}$ (bottom, solid line)

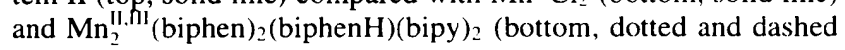
line). is $\sim 13 \mathrm{eV}$ for Fe complexes, a detector with $10^{3}$ energy resolution would be sufficient for measuring spin-polarized spectra. The development of ultra-high-energy-resolution detectors, such as the bolometer (Le Gros et al., 1995) and the superconducting tunnel junction detector (Frank et al., 1996), may eventually eliminate the whole crystal spectrometer for spin-polarized studies. It is, however, essential to have spectrometer resolution which is better than $1 \mathrm{eV}$ in order to utilize the chemical shifts $(\sim 1 \mathrm{eV})$ in mixed-valence and mixed-spin-state clusters for siteselective XAS.

\section{Conclusions}

The first high-resolution fluorescence spectra of metalloproteins have been made possible by the development of a multiple-crystal fluorescence spectrometer. The focus images of multiple form-bent crystals confirm that all diffracted beams are brought to a common focus within a region $3.8 \mathrm{~mm}$ (vertical) $\times 8.5 \mathrm{~mm}$ (horizontal) on the detector. The strengths of high-resolution fluorescence metalloprotein studies are both site and spin selectivity and can complement the structural information obtainable from other spectroscopies. In addition, the spin-polarized near-edge structure provides a new measure of the spin density. The current spectrometer has sufficient sensitivity for dilute protein experiments. With the anticipated orders of magnitude improvement in photon flux and spectrometer efficiency, site-selective and spin-polarized EXAFS can become a powerful probe of multinuclear proteins in the near future.

We would like to thank C. R. Randall and Professor Larry Que (University of Minnesota. Minnesota) for the $\left(\mathrm{NEt}_{4}\right)\left[\mathrm{FeCl}_{4}\right]$ sample, $\mathrm{H}$. Huang and Professor $\mathrm{M}$. Adams (University of Georgia, Athens) for the P. furiosus rubredoxin sample, and P. S. de Marois and Professor J. E. Penner-Hahn (University of Michigan, Ann Arbor) for the Photosystem II sample. This work was funded by the National Institutes of Health (GM-48145) and the Department of Energy, Office of Health and Environmental Research. The National Synchrotron Light Source and the Stanford Synchrotron Radiation Laboratory are supported by the Department of Energy, Office of Basic Energy Sciences.

\section{References}

Åberg, T. \& Tulkki, J. (1985). Atomic Inner-Shell Physics, edited by B. Crasemann. New York: Plenum.

Berman, L. E., Hastings, J. B., Oversluizen, T. \& Woodle, M. (1992). Rev. Sci. Instrum. 63, 428-432.

Blake. P. R., Park, J.-B., Bryant, F. O., Aono, S., Magnuson, J. K., Eccleston, E., Howard, J. B., Summers, M. F. \& Adams, M. W. W. (1991). Biochemistry, 30, 10885-10895.

Brennan. S. \& Cowan, P. L. (1992). Rev. Sci. Instrum. 63, $850-853$. 
Carra, P., Fabrizio, M. \& Thole, B. T. (1995). Phys. Rev. Lett. 74, 3700-3703.

Cowan, P. L. (1994). Resonamt Anomalous X-ray Scattering, edited by G. Materlik, C. J. Sparks \& K. Fischer, pp. 449-472. Amsterdam: North-Holland.

Debenham, M. J., Hao, Q., Hasnain, S. S., Dodd, F. E., Abraham, Z. H. L. \& Eady. R. R. (1996). J. Synchrotron Rad. 3, 14-19.

Eisenberger, P., Platzman, P. M. \& Winick, H. (1976a). Phys. Rev. Lett. 35, 623-626.

Eisenberger, P., Platzman, P. M. \& Winick, H. (1976b). Phys Rev. B, 31, 2377-2380.

Ekstig, B., Källne, E., Noreland. E. \& Manne, E. (1970). Phys. Scr. 2, 38-44.

Frank, M., Mears, C. A., Labov, S. E., Azgui, F., Lindeman, M. A., Hiller, L. J. \& Netel, H. (1996). Nucl. Instrum. Methods, 370, 41-43.

George, S. J., Lowery, M. D., Solomon, E. I. \& Cramer, S. P. (1993). J. Am. Chem. Soc. 115, 2968-2969.

George, S. J., van Elp, J., Chem. J., Ma, Y., Chen, C. T., Park, J.-B., Adams, M. W. W., Searle, B. G., de Groot, F. M. F., Fuggle, J. C. \& Cramer, S. P. (1992). J. Am. Chem. Soc. 114, 4426-4427.

Ghanotakis, D. F., Demetriou, D. M. \& Yocum, C. F. (1987). Biochim. Biophys. Acta, 891, 15-21.

Gill, N. S. (1961). J. Chem. Soc. (18), 3512.

Groot, F. M. F. de (1996). Phys. Rev. B, 53, 7099-7110.

Groot, F. M. F. de, Fontaine, A., Kao, C. C. \& Krisch, M. (1994). J. Phys. Cond. Matter, 6, 6875-6884.

Groot, F. M. F. de, Pizzini, S., Fontaine, A., Hämäläinen, K., Kao, C. C. \& Hastings, J. B. (1995). Phys. Rev. B, 51, 1045-1052.

Grush M. M., Christou, G., Hämäläinen, K. \& Cramer, S. P. (1995). J. Am. Chem. Soc. 117, 5895-5896.

Grush, M. M., de Marois, P. S., Daly, K., Penner-Hahn, J. E. \& Cramer, S. P. (1997). In preparation.

Grush, M. M., Horne, C. R. \& Cramer. S. P. (1997). In preparation.

Hämäläinen, K., Kao, C. C., Hastings, J. B., Siddons, D. P., Berman. L. E., Stojanoff, V. \& Cramer, S. P. (1992). Phys. Rev. $B, 46,14274-14277$.

Hämäläinen, K., Siddons, D. P., Hastings, J. B. \& Berman, L. E. (1991). Phys. Rev. Lett. 67, 285()-2853.

Hoyer, E., Bahr, C., Chan, T., Chin, J., Elioff, T., Halbach, K., Harnett, G., Humphries, D., Hunt. D., Kim, K.-J., Lauritzen, T., Lindel, D., Shirley, D., Tafelski. R., Thompson, A., Cramer, S., Eisenberger, P., Hewitt, R., Stöhr, J., Boyce, R., Brown, G., Golde, A., Gould, R., Hower, N.. Lindau, I., Winick, H., Yang, J., Harris, J. \& Scott, B. (1983). Nucl. Instrum. Methods, 208, $117-125$

Kirby, J. A., Robertson, A. S., Smith, J. P., Thompson, A. C., Cooper, S. R. \& Klein, M. P. (1981). J. Am. Chem. Soc. 103, 5529-5537.

Kobayashi, K., Maruyama, H., Maeda, H., Iwazumi, T., Kawata, H. \& Yamazaki, H. (1995). Physica B, 209, 779-780.

Krause, M. (1979). J. Phys. Chem. Ref. Data, 8, 307-327.

Krisch. M. H., Kao, C. C., Sette, F.. Caliebe, W. A., Hämäläinen, K. \& Hastings, J. B. (1995). Phys. Rev. Lett. 74, 4931-4934.

Kusunoki, M., Ono, T., Inoue, Y., Susuki, M., Uchara, A., Matsushita, T. \& Oyanagi, H. (1991). X-ray Absorption Fine Structure, edited by S. S. Hasnain, pp. 174-177. Chichester: Ellis Horwood.

Kusonoki, M., Ono, T., Matsushita, T., Oyanagi, H. \& Inoue, Y. (1990). J. Biochem. 108, 560-567.
Le Gros, M.. Silver, E., Schneider, D., McDonald, J., Bardin, S., Schuch, R., Madden, N. \& Beeman, J. (1995). Nucl Instrum. Methods, 357, 110-114.

Lindh, A. E. \& Lundquist, O. (1924). Ark. Mater. Astronom. Fys. 18, 14.

Marenkov, S. O. (1994). Handbook of Partial Attenuation Coefficients of Characteristic $X$-ray Radiation, edited by $\mathrm{O}$. S. Marenkov. New York: Nova Science Publishers.

Mori, A., Kayanuma, Y. \& Kotani, A. (1991). Prog. Theor. Phys. 106, 75-93.

Murphy, L. M., Dobson, B. R., Neu, M., Ramsdale C. A., Strange, R. W. \& Hasnain, S. S. (1995) J. Synchrotron. Rad. 2, 64-69.

Nordgren, J., Bray, G., Cramm, S., Nyholm, R., Rubensson, J.-E. \& Wassdahl, N. (1989). Rev. Sci. Instrum. 60, 1690-1696.

Peng, G., de Groot, F. M. F., Hämäläinen, K., Moore, J. A., Wang, X., Grush, M. M, Hastings, J. B., Siddons, D. P., Armstrong, W. H., Mullins, O. C. \& Cramer, S. P. (1994). J. Am. Chem. Soc. 116, 2914-2920.

Peng, G., Wang, X., Randall, C. R., Moore, J. A. \& Cramer, S. P. (1994). Appl. Phys. Lett. 65, 2527-2529.

Penner-Hahn, J. E., Fronko, R. M., Pecoraro, V. L., Yocum, C. F., Betts, S. D. \& Bowlby, N. R. (1990). J. Am. Chem. Soc. 112, 2549-2557.

Riggs, P. J., Mei, R., Yocum, C. F. \& Penner-Hahn, J. E. (1992). J. Am. Chem. Soc. 114, 10650-10651.

Sauer, K., Yachandra, V. K., Britt. R. D \& Klein, M. P. (1992). Mn Redox Enzymes, edited by V. L. Pecoraro, pp. 141-175. New York: VCH Publishers.

Schlacter, A. S. (1994). New Directions in Research with ThirdGeneration Soft X-ray Synchrotron Light Sources, edited by A. S. Schlacter \& F. J. Wuilleumier, pp. 1-22. Dordrecht: Kluwer Academic Publishers.

Schulke, W. \& Nagasawa, H. (1984). Nucl. Instrum. Methods, 222, 203-206.

Schütz, G., Wagner, W., Wilhelm, W., Kienle, P., Zeller, R., Frahm, R. \& Materlik, G. (1987). Phys. Rev. Lett. 58, 737-740.

Skytt, P., Guo, J. H., Wassdahl, N. \& Nordgren, J. (1995). Phys. Rev. A, 52, 3572-3576.

Smith, G. C. \& Yu, B. (1995). IEEE Trans. Nucl. Sci. 42, 541-547.

Sparks, C. J. Jr (1974). Phys. Rev. Lett. 33, 262-265.

Stojanoff, V., Hämäläinen, K., Siddons, D. P., Hastings, J. B., Bermab, L. E., Cramer, S. P. \& Smith, G. (1992). Rev. Sci. Instrum. 63, 1125-1127.

Tsiotis, G., McDermott, G. \& Ghanotakis, D. (1996). Photosynth. Res. 50, 93-101.

Udagawa, Y., Hayashi, H., Tohji, K. \& Mizushima, T. (1994). J. Phys. Soc. Jpn, 63, 1713-1720.

Wang, X. \& Cramer, S. P. (1997). In preparation.

Wang, X., Cramer, S. P., Zang, Y., Que, L. Jr, Aunodoxin, A. \& Rehr, J. (1997). In preparation.

Wang, X., Huang, H., Adams, M., Kotani, A. \& Cramer, S. P. (1997). In preparation.

Wang, X., Randall, C. R., Peng, G. \& Cramer, S. P. (1995). Chem. Phys. Lett. 243, 469-474.

Williams, G. P. (1986). X-ray Data Booklet, edited by D. Vaughan. University of California, Berkeley, USA.

Winick, H. (1995). J. Electron Spectrosc. 75, 1-8.

Yachandra, V. K., DeRose, V. J., Latimer, M. J., Mukerji, I., Sauer, K. \& Klein, M. P. (1993). Science, 260, 675-679.

Zachariasen, W. H. (1945). Theory of X-ray Diffraction in Crystals, edited by W. H. Zachariasen. New York: Dover Publications. 